\title{
ESTUDO EXPLORATÓRIO DOS ATENDIMENTOS EM UNIDADE DE EMERGÊNCIA EM SALVADOR - BAHIA
}

\author{
Peter Christian Jacobs*, Ediriomar Peixoto Matos
}

Trabalho realizado no Hospital São Rafael - Monte Tabor, Centro Ítalo-Brasileiro de Promoção Sanitária, Salvador, BA.

RESUMO
OBJETIVO. Analisar variáveis relativas aos atendimentos realizados em unidade de emergência que aplica protocolos.

Métodos. Objeto da análise: pacientes admitidos no serviço de emergência do Hospital São Rafael, Salvador, Bahia, de I de junho de 2000 a 3 I de maio de 200I. Estudo das variáveis idade, sexo, procedência, data, hora, especialidade médica, doença, complexidade e tempo de permanência.

Resultados. Dos pacientes estudados, 35,4\% tinham até 14 anos de idade; $86,11 \%$ dos atendimentos ocorreram das $8 \mathrm{~h}$ às $22 \mathrm{~h} 59$ 78,96\% de todos os atendimentos foram consultas seguidas de alta; $17,86 \%$ necessitaram de observação. Desses, $10,37 \%$ tiveram alta e 7,49\% foram internados. Permanência média de 282,9 minutos $( \pm 242,54)$. O grupo de CID10 "R" foi mais freqüente (29,5\%). As três principais causas de atendimento foram febre, diarréia e dispnéia. Dos procedentes de até $5 \mathrm{~km}$ do hospital, $12 \%$ necessitaram de observação e $5,8 \%$ de internamento. Dos procedentes de mais de $15 \mathrm{~km}$, $20,23 \%$ necessitaram de observação e $12,63 \%$ de internamento.

*Correspondência: Rua Sgt. Walmir Bannach, Qd. 24, no 26, Salvador, BA, Cep: $41650-330$ peter@atarde.com.br
ConclusöEs. A maioria dos pacientes que procuram a unidade de emergência necessita de atendimentos de baixa complexidade, principalmente quando procedem de bairros próximos ao hospital.

UNITERMOS: Emergência. Planejamento. Tempo de permanência. Protocolos. Epidemiologia.

\section{INTRODUÇÃO}

Os serviços prestados nas unidades de emergência estão relacionados ao grau de desenvolvimento e organização da sociedade, aspectos demográficos e culturais, estrutura e recursos governamentais. Em muitos países, há uma preocupação cujo foco principal são os serviços de emergência e a assistência ${ }^{1,2,3}$. Muitos modelos assistenciais existem e a reavaliação constante se faz necessária ${ }^{4,5,6,7}$. Ao planejar a assistência às situações de urgência e emergência é necessário ter o conhecimento do quê, com qual gravidade e a quem se atende ${ }^{8}$, quanto tempo se usa para estes atendimentos e o destino dos pacientes ${ }^{9,10}$.

O objetivo deste trabalho éa análise de um serviço de emergência de um hospital terciário, localizado na cidade de Salvador, Bahia, com a intenção de observar a quem se atende, com que tipo de doença, quanto tempo isto demora, de onde vem e qual o seu destino.

\section{Métodos}

Descrição do hospital: O Hospital São Rafael está localizado em Salvador, capital do Estado da Bahia, Brasil. É um dos seis grandes hospitais da região metropolitana de Salvador. Situa-se a 15 quilômetros do aeroporto e da rodoviária e a ele se tem acesso rápido por avenidas largas de diversos pontos da cidade e das rodovias interestaduais. Dispõe de 350 leitos, sendo 13 de UTI geral, nove de UTI cardíaca, oito de UTI pediátrica, 12 de semi-intensiva geral e oito de semi-intensiva especializada em doenças do aparelho digestivo. Dispõe de dois centros cirúrgicos, com um total de I 0 salas de cirurgia. Anexo ao hospital há um prédio de consultórios para atendimento ambulatorial. Dentre os serviços que existem no hospital, há os de oncologia e o de hemodiálise. Não dispõe de serviço de maternidade.
Nele ocorrem procedimentos de alta complexidade. De acordo com - Regulamento Técnico dos Sistemas Estaduais de Urgência e Emergência", seria uma Unidade Hospitalar de Referência em Atendimento às Urgências e Emergências do tipo III.

Descrição da unidade de emergência: A unidade de emergência do Hospital São Rafael dispõe de 17 leitos de observação para adultos e sete leitos de observação para crianças. Estão de plantão, 24 horas por dia, sistematicamente: um cardiologista, um clínico, dois cirurgiões, um ortopedista, dois pediatras e um radiologista. Estão de plantão, das $7 \mathrm{~h}$ às $19 \mathrm{~h}$, um intensivista e um neurologista. Estão de sobreaviso, com tempo médio de chegada ao hospital em 40 minutos, especialistas em: neurologia, neuropediatria eneurocirurgia, cirurgia buco-maxilo-facial, cirurgia de cabeça e pescoço, cirurgia plástica, cirurgia de tórax, cirurgia cardíaca, cirurgia vascular, cirurgia da coluna, cirurgia geral, urologia, ginecologia, pneumologia, endocrinologia, nefrologia, oftalmologia, otorrinolaringologia, infectologia e hemodinâmica. Estão à disposição para uso 24 horas: laboratório, tomografia computadorizada helicoidal, em horário administrativo (das $8 \mathrm{~h}$ às $18 \mathrm{~h}$ ), exames cintilográficos e ressonância nuclear magnética. A unidade de emergência não dispõe de ambulâncias para atendimento pré-hospitalar e transferência inter-hospitalar, mas recebe pacientes trazidos por serviços de ambulâncias. Não há rotina de triagem por médicos ou por enfermeiras. O tempo médio dos exames de laboratório, da coleta ao resultado, é de cerca de 2 horas e 30 minutos (I50 minutos).

Rotinas de atendimento: Os atendimentos são conduzidos tendo como guia protocolos de decisão ${ }^{12}$, em uso desde | $99 \mid$, para investigação propedêutica e para tratamento de acordo com as doenças. 
Os pacientes são admitidos por diversas vias: demanda espontânea, trazidos por serviços de ambulância e referenciados de outros hospitais da capital e do interior do Estado. Quando procuram o setor e não há risco iminente à vida são os pacientes ou os acompanhantes inquiridos pelo funcionário técnico administrativo da recepção quanto à queixa que os trouxe por meio de perguntas simples, seguindo tabela escrita preparada pelo coordenador médico. De acordo com a resposta há o direcionamento para um dos cinco especialistas de plantão 24 horas (clínico, cirurgião, pediatra, ortopedista eneurologista). Pacientes com idade inferior a I 4 anos são encaminhados sistematicamente para atendimento pediátrico. Aos pacientes com idade entre 14 e 18 anos é dado o direito de escolha entre 0 atendimento por pediatra ou clínico. Se, entretanto, o paciente ou o acompanhante verbalizam preferência para atendimento esta é acatada. Trauma em adultos, dor abdominal, hemorragia digestiva alta ou baixa, cólica nefrética, doenças ginecológicas, abscessos, corpos estranhos retidos e trauma ocular são atendidos primariamente pelo cirurgião. Febre, diarréias, doenças cardiovasculares e respiratórias de adultos são conduzidas por clínicos e cardiologistas. Entorses, luxações e fraturas, por ortopedistas. Doenças cerebrovasculares por neurologistas. Os traumas em crianças são conduzidos pelos pediatras. Caso algum paciente seja encaminhado de forma equivocada, os médicos o reconduzem a quem de direito.

Pacientes em risco iminente à vida são atendidos imediatamente pelo profissional mais próximo que, durante as primeiras medidas, identifica a queixa principal e o possível diagnóstico, solicitando que 0 profissional da especialidade mais a fim conduza o caso. Em situação de sobrecarga de demanda para uma determinada especialidade, os demais médicos assumem parte dos pacientes, procurando afinidades com a doença e/ou a faixa etária.

Os profissionais da emergência são treinados para estabilizar, diagnosticar e definir a necessidade de internamento o mais cedo possível.

O serviço gera, em média, $54 \%$ do total de internamentos hospitalares eletivos ou de emergência.

Descrição do estudo: $O$ estudo compreende pacientes atendidos de 0 h de I de junho de 2000 às 23 h59 de 3 I de maio de 200 I na unidade de emergência do Hospital São Rafael.

As variáveis idade, sexo, procedência (município e bairro) foram fornecidas verbalmente pelo paciente e digitadas na sua ficha informatizada, e estes dados ficaram então armazenados no banco de dados do hospital (sistema operacional SISA®). Cada paciente recebe um número único de prontuário, que o acompanha por toda a vida hospitalar e, cada vez que volta ao hospital, recebe um número seqüencial encerrado às $23 \mathrm{~h} 59$ de todo o dia 31 de dezembro e reiniciado anualmente com sequêencia que 0 identifica a cada ano isoladamente. A data e a hora são registradas automaticamente. $A$ especialidade do primeiro atendimento (clínica médica, cirurgia geral, ortopedia, pediatria eneurologia) é digitada pelo funcionário da recepção, inserida no banco de dados, e é modificada caso tenha havido encaminhamento equivocado. Para codificação das doenças foi utilizada a Classificação Internacional de Doenças e Problemas Relacionados à Saúde na sua décima revisão $(C I D-I 0)^{13}$. Durante $o$ atendimento inicial pelo médico, este registra manualmente na ficha a suspeita diagnóstica, o código CID-I0 da doença, a hora da alta e o destino do paciente. O código CID-I0, a hora da alta, destino do paciente (alta após consulta, consulta com observação e alta, consulta com observação e internamento) são inseridos no sistema operacional após a alta e mantidos no banco de dados. O tempo de permanência na unidade de emergência é calculado pelo computador quando da solicitação de dados ao centro de processamento de dados.

É denominado "observação" o período de tempo que transcorre do término da consulta inicial (que não resultou em uma alta) até uma alta hospitalar ou até a internação em unidade de internação aberta ou fechada (unidades de tratamento intensivo e semi-intensivo). Neste período, o paciente permanece num dos leitos de observação na unidade de emergência.

Todos os dados ficam armazenados no banco de dados do hospital - sistema operacional SISA ${ }^{\circledR}$, e foram obtidos mediante solicitação de levantamento de dados ao centro de processamento de dados do Hospital São Rafael.

As variáveis foram avaliadas individualmente com relação à sua freqüência, medidas de posição e de dispersão. Foram, então, feitos cruzamentos das variáveis entre si para investigação de tendências.

Para as análises comparativas com a distribuição demográfica, foram considerados os dados do censo do IBGE de 2000, tanto nas faixas de idade quanto na distribuição por gênero.

Para análise estatística, foram utilizados os programas de computador Microsoft Excel ${ }^{\circledR}$ e SPSS ${ }^{\circledR}$ "for Windows". Para testes de significância, foi utilizado o teste do Qui-quadrado de Pearson, considerando-se para a rejeição da hipótese nula um $\alpha \leq 5 \%(p \leq 0,05)$.

\section{Resultados}

Dos 59.35 I pacientes registrados como atendidos de 0 h de I de junho de 2000 a $23 \mathrm{~h} 59$ de 31 de maio de 200I, 57.662 foram analisados e I.689 foram excluídos. Os critérios de exclusão foram: ausência de digitação do CID-I0, ausência de digitação de sexo, especialidade digitada diferente das cinco especialidades básicas de plantão 24 horas, cruzamento do sexo com classificação pelo CID-I0 incompatível (exemplo: doença prostática em mulheres) e idade maior que I0I,25 anos em pacientes atendidos pela pediatria.

Resultados gerais: Dos 57.662 pacientes analisados, 53,1\% eram do sexo feminino e $46,9 \%$ do sexo masculino. A distribuição por faixa etária foi $35,4 \%$ até 14 anos de idade, $28,5 \%$ entre 15 e 34 anos, $30,4 \%$ entre 35 e 64 anos e $5,7 \%$ com idade superior a 65 anos.

Observou-se um número menor de atendimentos no mês de setembro e um número maior de atendimentos nos meses de janeiro, março e maio. Durante a semana, há um maior número de atendimentos às segundas-feiras. Os atendimentos ao longo do dia estão assim distribuídos: $86,2 \%$ acontecem entre as $8 \mathrm{~h}$ e as $22 \mathrm{~h} 59$, com picos de

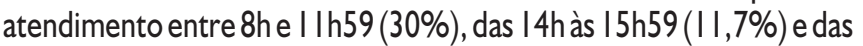
$19 \mathrm{~h}$ às $20 \mathrm{~h} 59$ ( $10,8 \%)$.

Quanto à procedência, observou-se que $96,35 \%$ dos pacientes vinham da região metropolitana, sendo que $86,5 \%$ do município de Salvador. Dos que moram no município de Salvador, avaliando-se a distância do bairro de procedência ao hospital, 30,35\% moram até $5 \mathrm{~km}$ de distância, $44,53 \%$ até $10 \mathrm{~km}, 63,53 \%$ até $15 \mathrm{~km}, 29,57 \%$ a mais de $15 \mathrm{~km}$, sendo que $6,9 \%$ não referiram o bairro de procedência. 
Tabela I - Relação de observações e internamentos por distância do hospital

\begin{tabular}{|c|c|c|c|}
\hline $\begin{array}{l}\text { Distância do bairro } \\
\text { ao hospital }\end{array}$ & $\begin{array}{l}\text { Percentual de observações em } \\
\text { relação ao número de atendimentos }\end{array}$ & $\begin{array}{l}\text { Percentual de internamentos em } \\
\text { relação ao número de atendimentos }\end{array}$ & $\begin{array}{l}\text { Relação de internamentos } \\
\text { por observações }\end{array}$ \\
\hline Até $5 \mathrm{~km}$ & $\begin{array}{l}3,6 \mid \\
\end{array}$ & 7,67 & 0.56 \\
\hline De 6 a $10 \mathrm{~km}$ & 16,88 & 9,79 & 0.57 \\
\hline De $1 /$ a $15 \mathrm{~km}$ & 19,00 & $|1,0|$ & 0,57 \\
\hline Mais de $15 \mathrm{~km}$ & 20,23 & 12,63 & 0,62 \\
\hline
\end{tabular}

Qui-quadrado de Pearson: $p \leq 0,05$ relativo às observações e internamentos de acordo com a distância

A distribuiç̧ão por especialidade dos plantonistas foi: clínica médica $(35 \%)$, pediatria $(32 \%)$, cirurgia (18\%), ortopedia (II\%) e neurologia (4\%).

Quanto ao tipo de atendimento, $78,96 \%$ foram consultas seguidas de alta, 10,37\% consultas com observação seguida de alta, 7,5\% consultas com observação seguida de internamento e $3,17 \%$ consultas seguidas de internamento.

Cruzamento das variáveis: Observou-se que a proporção de observações e internamentos se mantém durante todos os dias da semana, independente do aumento do número de consultas totais às segundas-feiras. Não ocorreu variação nos dias da semana quanto à especialidade do atendimento, também.

Avaliando se a procedência influenciaria no tipo de atendimento, observou-se que quanto mais perto do hospital a procedência, maior o número de consultas seguidas de alta sem observação, mas que a relação de internamentos por pacientes observados não sofria influência da procedência (Tabela I).

A distribuição por faixa etária demonstrou uma desproporção na progressão da relação atendimentos / número de habitantes nas faixas de um a nove anos, que é mais alta, de 10 a 34 anos, que é mais baixa. Avaliando-se a proporção de observações e internamentos por faixa etária, observamos que na faixa etária de um a quatro anos ocorre a menor proporção de observações por número de consultas (II,9\%)e que, de forma progressiva, esta proporção aumenta mais claramenteà partir dos 30 anos de idade (17,35\%) atéatingir $49,34 \%$ nos pacientes com 80 anos ou mais.

Assim também evolui a proporção de internamentos pelo número de consultas, a mais baixa entre um e nove anos de idade (6,5\%) com tendência ascendenteà partir dos 40 anos de idade (I2,34\%) até atingir os $48,33 \%$ nos pacientes de 80 anos ou mais.

O tempo de permanência geral na unidade de emergência foi em média de 282,9 minutos ( $\pm 242,54$ ), que equivale a 4 horas e 42 minutos. Este tempo varia de acordo com a especialidade do atendimento, sendo mais baixo na pediatria, com média de 253,79 minutos $( \pm 225,4 \mathrm{I})$, e mais alto na neurologia, com média de $33 \mathrm{I}, 48$ minutos $( \pm 256,43)$.

De acordo com o tipo do atendimento, o tempo de permanência evolui de 236,47minutos ( $\pm 198,07)$, nas consultas seguidas de alta, passando por 257,40 minutos $( \pm 227,76)$ nas consultas seguidas de internamento, 463,20 minutos ( $\pm 270,83$ ) - 7 horas e 43 minutos - nas consultas com observação e alta, e até 533,24 minutos $( \pm 342,77)$ quase 9 horas - nas consultas seguidas de observação e internamento.

Quando investigamos quais as causas mais freqüentes de atendimento na unidade de emergência codificada pelo CID- I0, observamos que os três grupos de doenças mais freqüentes são: os dos grupos " $R$ " (sintomas, sinais e achados anormais de exames clínicos e laboratório não classificados em outra parte); $28,5 \%$, "]" (doenças do aparelho respiratório), 16,4\%; " "S" (lesões, envenenamento e algumas outras conseqüências de causas externas), $11 \%$.

Independente de gerar observação ou internamento, as causas mais freqüentes de atendimento foram febre $(5,6 \%)$, diarréia e gastroenterite de origem infecciosa presumível $(4,5 \%)$, dispnéia (4, I\%), hipertensão essencial ( $4 \%$ ) e dor abdominal (3,9\%). O maior número de observações é causado por doenças do grupo "R", seguidas do grupo "I" (doenças do aparelho circulatório e cérebro-vascular) e do grupo "A" (algumas doenças infecciosas e parasitárias), e o maior número de internações é causado por doenças do grupo "R", seguidas do grupo "l" e então pelo das do grupo "K" (doenças do aparelho digestivo). Mas as neoplasias são responsáveis pelo maior número relativo de observações $(54,97 \%)$ e de internamentos $(70,17 \%)$ por número de pacientes atendidos.

Quando avaliados não por grupo mas por doença específica, as maiores geradoras de períodos de observação são abdome agudo, outras dores abdominais, diarréia aguda e hipertensão essencial.

As doenças que mais levam ao internamento são, em ordem decrescente: dispnéia, hipertensão essencial, outras dores abdominais, diarréia, angina instável e abdome agudo.

As causas mais freqüentes de internamento sem observação são: hipertensão essencial, dispnéia, angina instável e acidente vascular cerebral.

Quando se explorou exclusivamente os dados relativos às causas externas, codificadas nos CID-10 "S", "T" (lesões, envenenamentos e algumas outras conseqüências de causas externas), e "W", " $X$ " e $Y$ " (causas externas de morbidade e mortalidade), observou-se que, embora exista um maior número de mulheres em relação aos homens, segundo a distribuição demográfica pelo IBGE 2000, nos pacientes atendidos na emergência há, nestes grupos, uma maior proporção de homens $(57,43 \%)$ em relação às mulheres $(42,57 \%)$.

Como achados adicionais ao estudo exploratório, notamos que as doenças do grupo CID-I0 assumem progressivamente as primeiras posições como causa de atendimento a partir dos 35 anos de idade, chegando à segunda mais freqüente a partir dos 45 anos de idade, abaixo apenas do grupo "R", e à primeira a partir dos 60 anos.

Ao investigar a distribuição ao longo do dia dos pacientes deste grupo CID-IO “I”, não se observou diferença com relação aos demais grupos de doenças, apresentando todos a mesma distribuição que acompanha o número geral de atendimentos: maior número de atendimentos pela manhã, das $8 \mathrm{~h}$ às I Ih59, com queda progressiva ao longo do dia. 


\section{Dıscussão}

A escolha de estudar pacientes que dão entrada nesta unidade de emergência ocorreu devido à importância deste conhecimento para estabelecer estratégias locais e fornecer subsídios para interessados no assunto na tomada de decisões quanto à política de saúde direcionada para estes atendimentos.

A distribuição dos pacientes estudados por sexo acompanhou aquela da população brasileira e da região metropolitana de Salvador no mesmo período estudado (IBGE, Censo 2000).

A maior proporção de atendimentos com relação ao número de habitantes de Salvador na faixa etária entre um e nove anos pode estar relacionada ao maior número de eventos que levam a um atendimento médico na primeira faixa, como febre, dispnéia e diarréia. Pode sinalizar, também, o uso da unidade de emergência em detrimento do consultório do pediatra ou a falta de outros locais para atendimento pediátrico nas proximidades da unidade de emergência. Na faixa de 10 a 34 anos, a população seria mais saudável e menos propensa a doenças.

Observou-se, na contagem mensal, um aumento do número de atendimentos em janeiro, março e maio. Em janeiro, o pico de atendimentos se deveu às doenças do grupo CID-10 " $A$ " (algumas doenças infecciosas e parasitárias), principalmente as gastrenterites de origem bacteriana presumível, e por rotavírus, e doenças CID-I0 "G" (doenças do sistema nervoso), tendo como causa principal o diagnóstico de "outras cefaléias", assim como as do CID-10 "T" (lesões, envenenamento e algumas outras conseqüências de causas externas). Isto pode estar relacionado à maior mobilidade da população no período de férias escolares e à mudança de hábitos alimentares neste período.

Em março e maio, houve um aumento do número de casos de doenças do grupo CID-I0 "B", em destaque as infecções virais não especificadas e as não especificadas caracterizadas por lesões da pele e membranas mucosas. O maior número de atendimentos em março poderia ser condicionado pelo início do período escolar e pelo carnaval, quando a possibilidade de contato interpessoal é maior, assim como por um período de chuvas intermitentes na região, o que poderia facilitar a disseminação de doenças infecto-contagiosas.

O maior número de atendimentos que ocorre às segundas-feiras pode estar relacionado à facilidade de acesso ao sistema, disponibilidade de médico sem marcação prévia para atendimento imediato, e ao acúmulo de "urgências" não resolvidas no fim de semana. Entretanto, seria de se esperar um aumento de consultas seguidas de alta, mas isto não ocorre. Não se observou, contudo este aumento de atendimentos na segunda-feira no estudo de Ortega et al. ${ }^{14}$.

A distribuição dos atendimentos ao longo do dia obedece ao ciclo natural de sono / viǵlia dos seres humanos, independente do grupo de doenças codificadas pelo CID-I0, seja por doença cardiovascular ou por trauma; achados também de Bardenheuer, na Alemanha.

No estudo de Bardenheuer sobre politraumatizados graves na Alemanha ${ }^{15}$, observou-se uma relação de 2,6 homens por mulher afetada, porém $56,7 \%$ dos pacientes eram vítimas de acidentes automobilísticos e esta exclusividade de atenção não foi objetivo do nosso trabalho. Mesmo assim, há uma predominância do sexo masculino nos atendimentos por causa externa $(57,43 \%)$.
A procedência sugere que a urgência define o hospital mais próximo como destino preferencial, ficando-se de se estabelecer o porquê do uso da UE por pacientes que procedem de bairros além de $15 \mathrm{~km}$ de distância.

O tempo de permanência varia de acordo com o tipo de atendimento: 282,9 minutos ( $\pm 242,54$ ) na avaliação geral, 492,6 minutos $( \pm 305,05)$ nos submetidos à observação e 533,2 minutos $( \pm 342,77)$ nos submetidos à observação e internamento. No estudo de Ortega et al. ${ }^{14}$ este tempo médio é de 288 minutos. No estudo de Quick ${ }^{16}, 158$ minutos naqueles admitidos pelos plantonistas sem aguardar a decisão de especialistas. Fica, entretanto, difícil estabelecer alguma comparação, pois neste último estudo não há relato se estes pacientes passaram por período de observação antes da decisão de internar, e em nenhum deles está relatado o tempo médio gasto com coleta e resultados de exames, que na sede deste estudo é de 150 minutos (duas horas e 30 minutos), o que por si só pode influenciar o tempo de permanência.

A proporção de internamentos com relação ao total de atendimentos é de $10,67 \%$, semelhante àquela do estudo de Quick ${ }^{16}(12,2 \%$ e $10,7 \%)$, bem menor que no estudo de Nikkanen na França' ${ }^{\prime}$ que é de $30 \%$, mas cuja estrutura de triagem é bem mais agressiva, condicionando a diminuição do mau uso da emergência, mas nada comparado aos I,5\% do estudo de Lateef et al. ${ }^{17}$.

A proporção de internamentos com relação ao número de atendimentos deste estudo é três vezes maior que a esperada para uma unidade de atendimento não hospitalar nível I, II ou III, descrita no Diário Oficial da União como resultado de observação destas unidades, nas normas da Portaria $n^{\circ} 2048$, de 5 de novembro de 2002, do Ministério da Saúde, Regulamento Técnico dos Sistemas Estaduais de Urgência e Emergência". Não há, entretanto, nesta Portaria uma norma equivalente para os hospitais, denominados "Unidades Hospitalares de Referência em Atendimentos às Urgências e Emergências", também classificados em três tipos, nem o quantitativo de pacientes que se espera que estas unidades atendam, de acordo com o número de leitos disponíveis, ou pelo número de habitantes da cidade ou região.

Há um aumento progressivo na proporção de observações e internamentos de acordo com a faixa etária do paciente, chegando a $48,32 \%$ e a $49,34 \%$, respectivamente, nos pacientes com 80 ou mais anos, o que seria esperado.

A utilização da codificação dos agravos à saúde pelo CID- 10 tem sua dificuldade relacionada à grande utilização do CID-I0 "R". Muitos dos sinais e sintomas podem ser causados por doenças de diferentes aparelhos com codificação mais específica, não "R". Estaria a codificação condicionada à postura dos médicos plantonistas em utilizar os códigos "genéricos", mais fáceis de lembrar? Quais os critérios que estes médicos utilizariam para codificar o que atendem neste ou naquele código?

Febre, diarréia e dispnéia são os três motivos principais de atendimento, mas, se somarmos os pacientes atendidos com os CID-10 "RI0.0" abdome agudo (aos atendidos por "RI0.4" outras dores abdominais), esta soma passaria a ser o principal motivo de atendimento observado neste estudo. Estes achados também ocorrem em lugares distantes como a China, onde no estudo de Lyu-Y et al. ${ }^{18}$ se observou que as duas queixas principais que levaram paciente à UE foram febre 
$(20,9 \%)$ e dor abdominal $(20,1 \%)$ ou em Singapura onde no estudo de Lateef-Fetal. ${ }^{17}$, também queixas abdominais responderam por $45,1 \%$ das observações.

A disponibilidade, rapidez e resolução para aqueles pacientes que moram mais próximos da unidade de saúde (os exames de laboratório, prontos em 150 minutos, métodos de imagem disponíveis 24 horas sem marcação prévia, etc.) e a ausência de procedimentos de triagem que encaminhem os pacientes para 0 ambulatório ou para outros serviços podem ser a causa de um maior número de consultas seguidas de alta naqueles que procedem de bairros mais próximos ao hospital. Isto, como em Ortega etal. ${ }^{14}$, é também um motivo de insatisfação com a demora nos picos de atendimento, assim como afeta pacientes que realmente necessitam de atendimento médico, mas optam pela evasão sem atendimento.

Com certeza, não tem este estudo condições de englobar todas as nuances dos atendimentos em uma unidade de emergência. Propõe, entretanto, com sua leitura, com as críticas à sua forma de abordar o assunto e com as perguntas que estimule, mais do que com eventuais respostas, que este assunto passe a ser uma preocupação de muitos, pois o trabalho em unidades de emergência e a assistência às pessoas agudamente enfermas precisam ser do conhecimento de todos. É necessário que não se crie expectativas excessivas quanto ao que se pode oferecer, não se ofereça nada de menos do que se deva oferecer, do ponto de vista técnico, nem se gaste demais, educando a todos para o exercício de uma medicina de urgência e emergência eficaz.

\section{Conclusões}

Espera-se que este estudo seja o primeiro de uma série que tenha como sede a unidade de emergência deste hospital.

Concluiu-se que a distribuição dos atendimentos por gênero é a mesma da população brasileira e da região metropolitana de Salvador.

A relação do número de atendimentos por número de habitantes nas faixas de idade de um a nove anos é maior e na faixa de idade de 10 a 34 anos é menor, e que $35,4 \%$ dos atendimentos na unidade de emergência são de pacientes com idade inferior a 14 anos.

Neste período, houve um maior número de atendimentos nos meses de janeiro, março e maio, e um menor número de atendimentos no mês de setembro. Não há, entretanto, como afirmar se isto pode ser extrapolado ao longo do tempo sem realizar estudo com maior duração.

O maior número de atendimentos é para a especialidade de clínica médica, seguida da pediatria, cirurgia, ortopedia e neurologia, o que pode ser condicionado pelo sistema de distribuição de doentes por especialista utilizado na unidade sede.

Há um maior número de atendimentos às segundas-feiras. Não há alteração na relação de observações e internamentos pelo número de consultas também nas segundas-feiras.

O número de atendimentos ao longo das 24 horas acompanha 0 ciclo de sono-viǵlia e 86, I I \% dos atendimentos ocorrem entre $8 \mathrm{~h}$ e $22 \mathrm{~h} 59$, sendo que $86,49 \%$ dos pacientes procedem de Salvador. Destes, $68,23 \%$ procedem de bairros até $15 \mathrm{~km}$ de distância do hospital. A relação de observações e internamentos por número de consultas é maior quanto mais longe do hospital mora o paciente. A maioria dos pacientes que procuram a unidade de emergência necessita de atendimentos de baixa complexidade, principalmente quando procedem de bairros próximos ao hospital.

Dos pacientes, $78,96 \%$ são submetidos a consulta, $17,86 \%$ a consulta e observação e 7,49\% a consulta, observação e internamento, e 3, I6\% deles são internados sem passar por período de observação.

Os três grupos de doenças CID-I 0 mais freqüentes são "R", "J"e "S", sendo que febre, diarréia e dispnéia são as causas mais freqüentes de atendimento na UE.

Quanto mais idade o paciente tem, maior a relação de observações e internamentos por consulta.

As principais causas de consulta seguida de observação são: abdome agudo, outras dores abdominais e diarréia. As principais causas de internamento são: dispnéia, hipertensão essencial e outras dores abdominais.

Há uma mudança nas causas mais freqüentes de atendimento de acordo com a faixa de idade: a partir dos 30 anos, há um aumento da frequêencia das doenças do aparelho circulatório (CID-I0 "I"), sendo que dos 45 em diante passa a ser a segunda e dos 60 anos em diante a primeira causa de atendimento.

Os I5 grupos de doenças mais freqüentes, codificadas pelo CID10 , têm distribuição semelhante ao longo do dia, inclusive as do grupo CID-IO "I", obedecendo os picos de atendimento.

Esteé um estudo descritivo do que ocorre na unidade de emergência do hospital sede. A coleta de dados é contínua e sua análise será aprofundada. Espera-se que possa ser feito em outras instituições, individualmente ou de forma multicêntrica, para fins de comparação, além de servir de apoio às decisões que visem à melhoria da qualidade dos atendimentos nas unidades de emergência, como se hospitaissentinela fossem.

Diante do que foi observado, sugere-se que nas unidades de emergência haja reforço de pessoal no horário das 8 hàs $22 \mathrm{~h} 59$; que se tente diminuir ao mínimo necessário o tempo de permanência na unidade; que haja um programa de educação da clientela visando à diminuição das consultas por motivos mais simples e um programa de treinamento baseado em dados estatísticos como estes aqui apresentados.

\section{Conflito de interesse: não há.}

\section{SUMMARY}

Exploratory study of an Emergency Unit in Salvador, BaHia, BraziL

OBJECTIVE. Analysis of patient care variables in an emergency unit that applies care protocols.

METHODS. Subjects: Patients admitted to the emergency unit of the São Rafael Hospital in Salvador, Bahia, Brazil, between July I, 2000 and May 3I, 200I. Study of the variables: age, gender, origin (city, district), admission date and hour, medical specialty, patient's disease (coded by ICDM- I0), destiny (discharge, observation and discharge, observation and hospital admission), length of stay.

RESULTS. $35.4 \%$ of the patients were under 14 years old. $86.1 / \%$ of the admissions took place from 08:00 am to $10: 59 \mathrm{pm} .78 .96 \%$ of all patients were discharged after consultation. $17.86 \%$ required 
observation. Of these, $10.37 \%$ were discharged and $7.49 \%$ were admitted to the hospital. Length of stay was on the average of 282.9 ( \pm 242.54$) \mathrm{min}$. The ICDM-10 " $R$ " group had a higher frequency, $(29.5 \%)$. The three main causes for emergency unit admissions were fever, diarrhea and dyspnea. $12 \%$ of those who came from a distance of up to $5 \mathrm{~km}$ required observation and $5.8 \%$ needed hospital admission. $20.23 \%$ of those that came from a distance farther than $15 \mathrm{~km}$ required observation and $12.63 \%$ needed hospital admission.

CONCLUSIONs. The majority of patients cared for in this emergency unit required low complexity care and the number increases when patients come to the hospital from a shorter distance. [Rev Assoc Bras 2005; 5 I (6): 348-53]

KEY WORDS: Emergency. Planning. Length of stay. Protocols. Epidemiology.

\section{RefERÊNCIAS}

I. Nikkanen HE, Pouges C, Jacobs LM. Emergency medicine in France. Ann Emerg Med I998;3I(I):II6-20.

2. Miro $O$. Revisitas no programadas en un servicio de urgencias de medicina hospitalario: incidencia y fatores implicados. Med Clin Barc 1999; II2(16):610-5.

3. Moecke H. Emergency Medicine in Germany. Ann Emerg Med 1998;3 I(I):III-5.

4. Steg A. Restructuring of emergency services: a safety imperative. Bull Acad Natl Med 1994; 178(8): 1475-92.

5. ACEP. American College of Emergency Physicians USA. Emergency Care Guidelines. Ann Emerg Med 1997;29(4):564-7I

6. Dick W. Structure, organization and capacity problems in emergency medical services, emergency admission and intensive care units. Zentralbl Chir 1994; I I9(10):673-82.

7. Kirsch TD, Holliman CJ, Hirshon JM, Doezema D. The development of international emergency medicine: a role for U.S. emergency physicians and organizations. SAEM International Interest Group. Acad Emerg Med 1997;4(10):996-100I.
8. Cioffi J. Recognition of patients who require emergency assistance; a descriptive study. Heart Lung 2000;29(4):262-8.

9. Cairnes CB, Garrison HG, Hedges JR, Schriger DL, Valenzuela TD. Development of new methods to assess the outcomes of emergency care. Ann Emerg Med I 998;3 I (2): I 66-7I.

I0. Soto-CamposJG, Rodriguez-Becerra E, Alvarez-Gutierrez FJ, ValenzuelaMateo F, Penafiel-Colas M, Castillo-GomezJ. Outpatient emergency care in pneumology. A method to alleviate pressure of hospital emergency services. Arch Bronconeumol 1997;33(6):268-7I.

II. Brasil. Portaria ${ }^{\circ} 2048$, de 05 de novembro de 2002. Diário Oficial da Uniãon $n^{\circ} 219: 32-54$.

12. Monte Tabor. Hospital São Rafael. Protocolos da unidade de emergência. $10^{a}$ ed. Salvador: Monte Tabor; 2003.

13. OMS. Organização Mundial de Saúde. Classificação estatística internacional de doenças e problemas relacionados à saúde. $10^{\mathrm{a}}$ rev. Brasília: OMS; 2000.

14. Ortega M, Esteban MJ, Miro O, Sanchez M, Milla J. Prospective study of patients who leave the emergency department before being seen by the physician. Med Clin Barc 2000; I I ( I): I 5-20.

15. Bardenheuer M, Obertacke U, Waydas C, Nast-Kolb D. Epidemiology of the severely injured patient. A prospective assessment of preclinical and clinical management. AG polytrauma of DGU. Unfallchirurg 2000; 103(5):355-63.

16. Quick G. Time analysis of consult service emergency department admission process compared with emergency medicine service admission process.J Emerg Med 1999; I 7(5):815-22.

17. Lateef-F, Anantharaman-V. The short-stay emergency observation ward is here to stay. Am J Emerg Med 2000; I 8(5):629-34.

18. Liu Y, Zhang B, Fu W, Li J, Singal B, Hamilton GC. A preliminary epidemiological study of the patient population visiting an urban ED in the Republic of China. Am J Emerg Med 1994; I 2(2):247-9.

Artigo recebido: 19/07/04 Aceito para publicaçãa: 25/07/05 